\title{
Broadband Mid-IR Fiber Supercontinuum Source for Hyperspectral Image Projection
}

\author{
L. Brandon Shaw, Rafael R. Gattass, and Jas S. Sanghera \\ Naval Research Laboratory, Code 5620, Washington, DC 20375 \\ Ishwar D. Aggarwal \\ Sotera Defense Solutions, Crofton, MD 21114 \\ Joseph P. Rice \\ National Institute of Standards and Technology, Gaithersburg, MD 20899
}

Testing, calibration, and validation of imaging sensors and imaging systems require bright sources of emission to simulate scene artifacts. Ideally, such sources are broadband or tunable over a wide wavelength region to provide spectral content to the scenes and/or to target the spectral response of the system. In the mid-infrared (IR), projection systems for sensor testing typically use globars, blackbodies, resistor arrays, optical parametric oscillators (OPO), or quantum cascade laser sources. Globars and resistor arrays are not bright enough to simulate high temperature artifacts which may be necessary for testing of some sensors and systems. OPO sources aand quantum cascade laser sources do not have the spectral coverage. To meet testing requirements for IR sensor systems, we have developed a broadband fiber based supercontinuum mid-IR source for projection systems..

The National Institute of Standards and Technology (NIST), Gaithersburg, MD, is developing a Hyperspectral Image Projector (HIP) for the testing, calibration, and system-level validation of multispectral and hyperspectral imaging sensors [1,2]. The system is capable of projecting scenes with specific spectral content at video frame rates. A schematic of the HIP system is shown in Fig. 1 below. The system is based upon two modified-commercial digital micromirror devices (DMDs) which are used in series to project the spectral content (DMD1) and the spatial content (DMD2) of the scene.

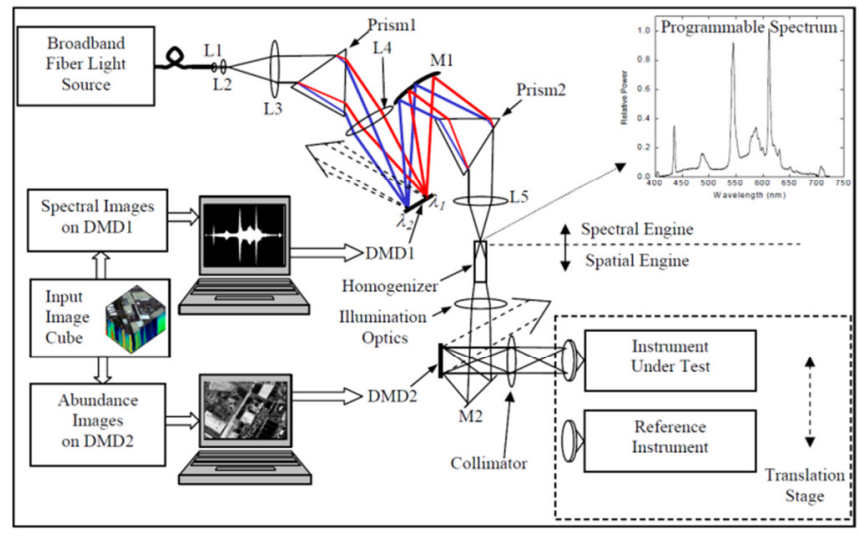

Figure 1. Schematic of the HIP system under development at NIST [2].

For the HIP system, Naval Research Lab is developing an IR fiber supercontinuum source [3]. Requirements for illumination of the HIP system are spectral power density of $-20 \mathrm{dBm} / \mathrm{nm}$ or higher, spectral flatness of $10 \mathrm{~dB}$ within the band of interest $(3 \mu \mathrm{m}$ to $5 \mu \mathrm{m})$, and continuous wave $(\mathrm{CW})$ or high repetition rate pulse trains. The repetition rate must be $\sim 1 \mathrm{MHz}$ or greater so that it far exceeds the temporal response of the system under test. The output diameter of the fiber can be no greater than $20 \mu \mathrm{m}$ in diameter to maintain the spectral resolution of the system.

The supercontinuum source is composed of a 2 meter length of single-mode $10 \mu \mathrm{m}$ core $/ 140 \mu \mathrm{m}$ clad $\mathrm{As}_{2} \mathrm{~S}_{3}$ fiber pumped with an amplified and Raman shifted $500 \mathrm{kHz}, 80 \mathrm{ps}$ Er mode-locked fiber laser. As the pump and generated supercontinuum fall within the normal dispersion regime of the fiber, the mechanism of supercontinuum is primarily cascaded Raman and self phase modulation. The use of a broadband pump with several cascaded 
Raman shifts convoluted with the Raman gain bandwidth of the fiber and spectral broadening due to self phase modulation results in a broadband flat continuous spectrum from $1.5 \mu \mathrm{m}$ to $5 \mu \mathrm{m}$ as shown in Fig. 2. Currently, spectral flatness is $10 \mathrm{~dB}$ from 1.9 to $4.4 \mu \mathrm{m}$ and $20 \mathrm{~dB}$ from 1.64 to $4.78 \mu \mathrm{m}$.

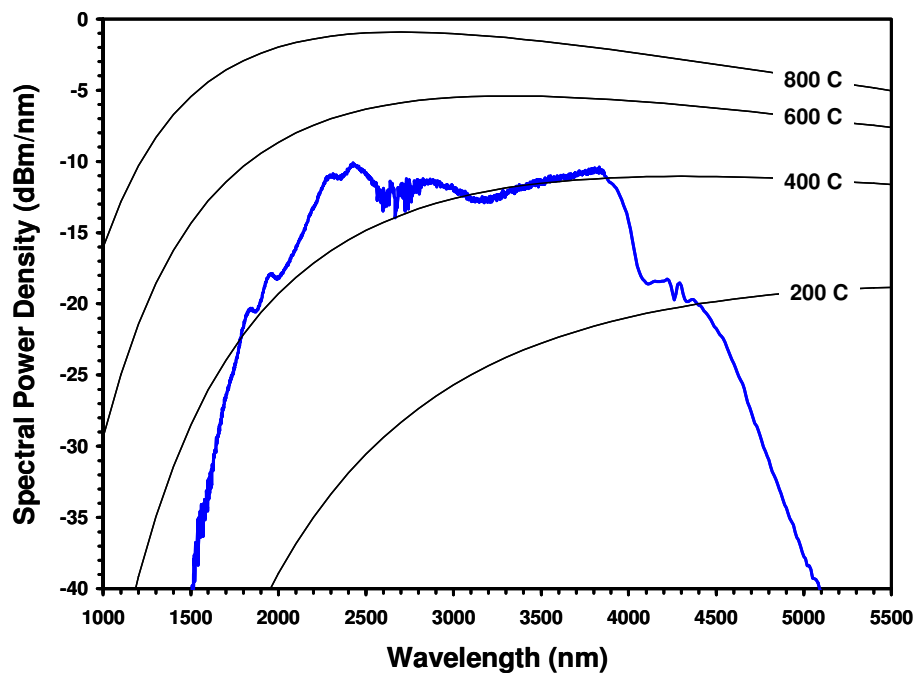

Figure 2. Mid-IR supercontinuum from 2 meter length $\mathrm{As}_{2} \mathrm{~S}_{3}$ fiber. The black temperature lines indicate equivalent blackbody temperature of a projected scene from the HIP system using the source.

In the HIP system, the power from the mid-IR supercontinuum is spread evenly over a $14 \mathrm{~mm} \times 10.5 \mathrm{~mm}$ DMD (DMD2 in Fig. 1) with $\mathrm{f} / 3$ divergence. Currently, the prototype source emits $\sim 140 \mathrm{~mW}$ of power within the spectral band. The estimated throughput of the source through the HIP is $\sim 9 \%$. Based on this, the HIP will project images with apparent temperatures of $400{ }^{\circ} \mathrm{C}$ to $500{ }^{\circ} \mathrm{C}$ in the mid-IR up to $\sim \mu \mathrm{m}$, where the spectrum of each image is programmable with an $8 \mathrm{~nm}$ spectral resolution. The control of not only the image, but also the spectrum of the image, is the key feature that distinguishes the HIP from other infrared scene projectors, and is what motivates the need for the high-brightness supercontinuum source. The spectral power density of an equivalent blackbody source is overlaid in black lines in Fig. 2. To further improve the source performance, we are in the process of scaling up power by increasing the repetition rate of the pump laser source from $500 \mathrm{kHz}$ to $5 \mathrm{MHz}$, resulting in an increase of 10 in average power. Also, the current supercontinuum bandwidth is limited by H-S impurity absorption in the $\mathrm{As}_{2} \mathrm{~S}_{3}$ fiber. By both decreasing the H-S content in the fiber and increasing the peak power of the pump source, we expect to increase the power beyond $4.0 \mu \mathrm{m}$. The goal is to project scenes with an apparent temperature of $>400{ }^{\circ} \mathrm{C}$ over the $3 \mu \mathrm{m}$ to $5 \mu \mathrm{m}$ band, while maintaining a spectral resolution of $8 \mathrm{~nm}$.

\section{Acknowledgements}

Funding for this work was provided by the Test Resource Management Center through the Science and Technology/Test and Evaluation Program.

\section{References}

[1] J.P. Rice, S.W. Brown, and J.E. Neira, Development of hyperspectral image projectors, Proceedings of SPIE, Vol. 6297 (2006)

[2] J.P. Rice, J. Niera, M. Kehoe, and R. Swanson, "DMD diffraction measurements to support design of projectors for test and evaluation of multispectral and hyperspectral imaging sensors, Proceedings of SPIE, Vol. 7210 (2009)

[3] L. B. Shaw, R.R. Gattas, J.S. Sanghera, and I.D. Aggarwal, "All-Fiber Mid-IR Supercontinuum Source from 1.5 to $5 \mu \mathrm{m}$," Proceedings of SPIE, Vol. 7914 (2011) 